\title{
Embedding into Rectilinear Spaces
}

\author{
H.-J. Bandelt, ${ }^{1}$ V. Chepoi, ${ }^{2}$ and M. Laurent ${ }^{3}$ \\ ${ }^{1}$ Mathematisches Seminar, Universität Hamburg, \\ Bundesstrasse 55, D-20146 Hamburg, Germany \\ ${ }^{2}$ Laboratoire de Biomathématiques, Université d'Aix Marseille II, \\ 27 Boulevard Jean Moulin, F-13385 Marseille cedex 5, France \\ aria@aix.pacwan.net \\ ${ }^{3}$ LIENS, Ecole Normale Supérieure, \\ 45 rue d'Ulm, F-75230 Paris cedex 05, France \\ monique@cwi.nl
}

\begin{abstract}
We show that the problem whether a given finite metric space $(X, d)$ can be embedded into the rectilinear space $\mathbb{R}^{m}$ can be formulated in terms of $m$-colorability of a certain hypergraph associated with $(X, d)$. This is used to close a gap in the proof of an assertion of Bandelt and Chepoi [2] on certain critical metric spaces for this embedding problem. We also consider the question of determining the maximum number of equidistant points that can be placed in the $m$-dimensional rectilinear space and show that this number is equal to $2 m$ for $m \leq 3$.
\end{abstract}

\section{Introduction}

Given a metric space $(X, d)$ let $\operatorname{dim}_{\ell_{1}}(X, d)$, or $\operatorname{dim}(X, d)$ for short, denote the smallest integer $m$ for which $(X, d)$ can be embedded into the $m$-dimensional rectilinear space, that is, $\mathbb{R}^{m}$ equipped with the $\ell_{1}$-distance. This number is called the $\ell_{1}$-dimension of $(X, d)$. The metric space $(X, d)$ is said to be critical if every proper subspace of $(X, d)$ has a smaller $\ell_{1}$-dimension than $(X, d)$. Critical metric spaces are essential for the determination of the order of congruence $c(m)$ of the $m$-dimensional rectilinear space. Here, $c(m)$ denotes the smallest integer such that an arbitrary metric space $(X, d)$ embeds into the $m$-dimensional rectilinear space if and only if the same holds for every subspace of $(X, d)$ on at most $c(m)$ points. The study of the parameter $c(m)$ is motivated by the following classical result of Menger [12]: A metric space $(X, d)$ embeds into the Euclidean space $\mathbb{R}^{m}$ if and only if the same holds for every subspace of $(X, d)$ on at most $m+3$ points. 
Hence, $c(1)=4$ by Menger's result. Bandelt and Chepoi [2] show that $c(2)=6$. This result was also established by Bonan-Hamada [4]. Malitz and Malitz [11] show that $c(m) \geq 2 m+1$ for all $m$. This bound was improved to $2 m+2$ by Schmerl in some unpublished letter (1992). Bandelt and Chepoi [2] assert that $c(m) \geq m^{2}$ for all $m$ odd. The proof for the latter assertion relies on the claim that the complete graph $K_{2 m+1}$ yields a critical metric space with $\ell_{1}$-dimension $m+1$. The proof for this fact is however not correct as it assumes that an embedding realizing the $\ell_{1}$-dimension is provided by the $d$-splits of $K_{2 m+1}$. We provide here another argument for demonstrating the inequality: $c(m) \geq m^{2}$ for $m$ odd. It is based on the analysis of critical metric spaces arising from the Cartesian product of two stars. Moreover, it also permits us to show that $c(m) \geq m^{2}-1$ for $m$ even, $c(3) \geq 10$. We also supply a proof for the assertion of [2] concerning the $\ell_{1}$-dimension of the complete graph up to dimension 3; namely, we show that the complete graph $K_{2 m+1}$ has $\ell_{1}$-dimension $m+1$ for $m \leq 3$.

\section{A Coloring Criterion for $\ell_{1}^{m}$-Embeddability}

In what follows we present some basic tools for studying the notion of $\ell_{1}$-dimension. For the sake of brevity we say that $(X, d)$ is $\ell_{1}^{m}$-embeddable if $(X, d)$ can be embedded into the $m$-dimensional rectilinear space. We first give an elementary characterization for $\ell_{1}^{m}$-embeddable metric spaces in terms of nested families of cuts, which can also be reformulated in terms of a coloring property of an associated hypergraph.

We begin with several observations concerning the structure of $\ell_{1}^{m}$-embeddable metrics, where $m \geq 1$ is an integer. A basic property that is used throughout this note is the fact that a metric space $(X, d)$ is $\ell_{1}$-embeddable if and only if $d$ can be decomposed as a positive combination of metrics associated with cuts (alias splits) $S, \bar{S}$ of $X$, where $\emptyset \neq S \subseteq X$ and $\bar{S}=X \backslash S$; namely,

$$
d=\sum_{\delta(S) \in \mathcal{S}} \lambda_{S} \delta(S)
$$

where $\lambda_{S}>0$ for all $\delta(S) \in \mathcal{S}$ and $\mathcal{S}$ is a family of cut metrics (or simply cuts) on $X$. Remember that $\delta(S)$ denotes the metric on $X$ taking value 1 for a pair of points $x \in S, y \in X \backslash S$, and value 0 otherwise. We let CUT(X) denote the cone generated by the cuts $\delta(S)$ for $S \subseteq X$; hence CUT $(X)$ consists precisely of the metrics on $X$ that are $\ell_{1}$-embeddable. When $d$ is $\ell_{1}^{m}$-embeddable then $d$ has such a decomposition with a special structure. We need some definitions in order to formulate it.

Given a family $\mathcal{S}$ of cuts on $X$, set

$$
\widetilde{\mathcal{S}}:=\{S, \bar{S} \mid \delta(S) \in \mathcal{S}\}
$$

the members of $\widetilde{\mathcal{S}}$ are the shores (or half-spaces) of the cuts in $\mathcal{S}$. The family $\mathcal{S}$ is said to be nested if the elements of $\widetilde{\mathcal{S}}$ can be ordered as $S_{1}, \ldots, S_{m}, \bar{S}_{1}, \ldots, \bar{S}_{m}$ in such a way that $S_{1} \subset S_{2} \subset \cdots \subset S_{m}$ (and thus, $\bar{S}_{m} \subset \cdots \subset \bar{S}_{2} \subset \bar{S}_{1}$ ). Given an integer $m \geq 1, \mathcal{S}$ is said to be $m$-nested if $\mathcal{S}$ can be partitioned into $m$ nested subfamilies. (Hence, 1-nested means nested.)

Two cuts $\delta(A)$ and $\delta(B)$ are said to form a incompatible pair if the four sets $A \cap B$, $\bar{A} \cap B, A \cap \bar{B}$, and $\bar{A} \cap \bar{B}$ are nonempty; that is, if no two sets among $A, \bar{A}, B, \bar{B}$ are 
comparable with respect to inclusion. Three cuts $\delta(A), \delta(B), \delta(C)$ are said to form an asteroidal triplet if there exist $\widetilde{A} \in\{A, \bar{A}\}, \widetilde{B} \in\{B, \bar{B}\}, \widetilde{C} \in\{C, \bar{C}\}$ such that $\widetilde{A}, \widetilde{B}, \widetilde{C}$ are pairwise disjoint. Hence, the family $\{\delta(A), \delta(B), \delta(C)\}$ is nested precisely if it does not contain any incompatible pair and does not constitute an asteroidal triplet. Let $\mathrm{H}(\mathcal{S})$ denote the hypergraph with node set $\mathcal{S}$ and whose edges are the incompatible pairs and asteroidal triplets, called the nesting hypergraph of $\mathcal{S}$. Then it can be easily verified that $\mathcal{S}$ is nested if and only if its nesting hypergraph $\mathrm{H}(\mathcal{S})$ is 1-colorable, i.e., has no edge. (A hypergraph is said to be $k$-colorable if its vertices can be colored with $k$ colors in such a way that no edge is monochromatic.) This follows from the fact that a family $\mathcal{S}$ is nested if and only if any three members of $\mathcal{S}$ form a nested subfamily. Therefore,

Remark 2.1. A family $\mathcal{S}$ of cuts is $m$-nested if and only if its nesting hypergraph $\mathrm{H}(\mathcal{S})$ is $m$-colorable.

We have the following easy description of $\ell_{1}^{m}$-embeddability.

Lemma 2.2. Let $(X, d)$ be a metric space. The following assertions are equivalent:

(i) $(X, d)$ can be embedded into $\ell_{1}^{m}$.

(ii) $d$ admits a decomposition of the form (2.1) where $\mathcal{S}$ is m-nested or, equivalently, whose nesting hypergraph $\mathrm{H}(\mathcal{S})$ is $m$-colorable.

(iii) $(X, d)$ can be isometrically embedded into the Cartesian product of $m$ weighted paths.

Proof. We first consider the case when $m=1$.

(i) $\Rightarrow$ (ii), (iii) Suppose that $d$ is $\ell_{1}^{1}$-embeddable. Then there exist scalars $u_{1}, \ldots, u_{n}$ such that $d(i, j)=\left|u_{i}-u_{j}\right|$ for all $i, j \in X$. Up to a reordering of the elements of $X$, we can suppose that $u_{1} \leq u_{2} \leq \cdots \leq u_{n}$. Then

$$
d=\sum_{i=1}^{n-1}\left(u_{i+1}-u_{i}\right) \delta(\{1, \ldots, i\}) .
$$

Then (ii) holds as the family $\{\delta(\{1, \ldots, i\}) \mid i=1, \ldots, n-1\}$ is obviously nested. Consider the path $P=(1, \ldots, n)$ with weight $w_{i, i+1}:=u_{i+1}-u_{i}$ on edge $(i, i+1)$ $(i=1, \ldots, n-1)$. Then the shortest path metric of the weighted path $P$ coincides with $d$, which shows (iii).

(iii) $\Rightarrow$ (i) Let $P=(1, \ldots, n)$ be a path with nonnegative weight $w_{i}$ on edge $(i, i+1)$ for $i=1, \ldots, n-1$. Then its path metric $d_{P, w}$ can be decomposed as $\sum_{i=1}^{n-1} w_{i} \delta(\{1, \ldots, i\})$.

(ii) $\Rightarrow$ (i) Suppose now that $d=\sum_{i=1}^{p} \alpha_{i} \delta\left(S_{i}\right)$, where $\alpha_{i}>0$ for all $i$ and $S_{1} \subset$ $S_{2} \cdots \subset S_{p}$. Define $u_{1}, \ldots, u_{n} \in \mathbb{R}$ by setting

$$
\begin{array}{rlrl}
u_{i} & :=0 & \text { for } \quad i \in S_{1}, \\
u_{i}:=\sum_{h=1}^{k} \alpha_{h} & \text { for } \quad i \in S_{k+1} \backslash S_{k}, \quad k=1, \ldots, m-1, \\
u_{i}:=\sum_{h=1}^{p} \alpha_{h} & \text { for } \quad i \in X \backslash S_{p} .
\end{array}
$$


Then it can be verified that $d(i, j)=u_{j}-u_{i}$ for all $i, j \in X$, which shows that $d$ is $\ell_{1}^{1}$-embeddable. The result for $m \geq 2$ now follows easily, since $d$ is $\ell_{1}^{m}$-embeddable if and only if $d$ can be decomposed as a sum $d=d_{1}+\cdots+d_{m}$, where all $d_{i}$ 's are $\ell_{1}^{1}$-embeddable.

It is not clear how to use this result for devising a polynomial algorithm permitting the recognition of $\ell_{1}^{m}$-embeddable metrics. The difficulty is that a metric in the cone CUT $(X)$ has, in general, many decompositions of the form (2.1) (in fact, an infinity if it is not $\ell_{1}$-rigid-see the definition below). However, in the case $m=1,2$, Bandelt and Chepoi [2] have observed that it is enough to check one special decomposition (namely, the one corresponding to a "total decomposition" of $d$ in the terminology of [3]). Moreover, they show that $\ell_{1}^{2}$-embeddability can be ensured by verifying all five- and six-point subspaces; that is, $c(2)=6$. This implies that $\ell_{1}^{2}$-embeddability can be checked in polynomial time; Malitz and Malitz [11] assert that this can be done in time $O\left(n^{3}\right)$ for a metric on $n$ points. We recall that, on the other hand, checking $\ell_{1}$-embeddability (i.e., embeddability into some rectilinear space $\mathbb{R}^{m}$ for unrestricted $m$ ) is NP-complete [10].

If a metric $d$ happens to have a unique decomposition of the form (2.1), then its $\ell_{1}$ dimension can be determined by inspection of the family $\mathcal{S}$ entering its decomposition (2.1); we shall see several such examples in Section 3 below.

A useful tool for studying the $\ell_{1}$-dimension is the additivity property for the $\ell_{1}$ dimension of a direct product of metric spaces, which we establish next. Recall that the direct product of two metric spaces $\left(X_{1}, d_{1}\right)$ and $\left(X_{2}, d_{2}\right)$ is the metric space $(X, d)$ where $X:=X_{1} \times X_{2}$ and

$$
d\left(\left(x_{1}, x_{2}\right),\left(y_{1}, y_{2}\right)\right):=d_{1}\left(x_{1}, y_{1}\right)+d_{2}\left(x_{2}, y_{2}\right)
$$

for $x_{1}, y_{1} \in X_{1}, x_{2}, y_{2} \in X_{2}$.

Lemma 2.3. Let $\left(X_{1}, d_{1}\right),\left(X_{2}, d_{2}\right)$ be two metric spaces, and let $(X, d)$ be their direct product. Then $\operatorname{dim}(X, d)=\operatorname{dim}\left(X_{1}, d_{1}\right)+\operatorname{dim}\left(X_{2}, d_{2}\right)$.

Proof. The inequality $\operatorname{dim}(X, d) \leq \operatorname{dim}\left(X_{1}, d_{1}\right)+\operatorname{dim}\left(X_{2}, d_{2}\right)$ follows by additivity of the $\ell_{1}$-metric. Conversely, assume that $(X, d)$ is $\ell_{1}^{m}$-embeddable. Then $d$ can be decomposed as $d=\sum_{\delta(S) \in \mathcal{S}} \lambda_{S} \delta(S)$, where $\mathcal{S}$ is a family of cuts on $X$ that is $m$-nested. Observe now that every cut $\delta(S)$ is of the form $\delta\left(A \times X_{2}\right)$ or $\delta\left(X_{1} \times B\right)$ for some $A \subset X_{1}$, $B \subset X_{2}$. This follows from the fact that $d$ satisfies the triangle equalities:

$$
\begin{aligned}
& d\left(\left(x_{1}, x_{2}\right),\left(y_{1}, y_{2}\right)\right)=d\left(\left(x_{1}, x_{2}\right),\left(y_{1}, x_{2}\right)\right)+d\left(\left(y_{1}, x_{2}\right),\left(y_{1}, y_{2}\right)\right), \\
& d\left(\left(x_{1}, x_{2}\right),\left(y_{1}, y_{2}\right)\right)=d\left(\left(x_{1}, x_{2}\right),\left(x_{1}, y_{2}\right)\right)+d\left(\left(x_{1}, y_{2}\right),\left(y_{1}, y_{2}\right)\right)
\end{aligned}
$$

for all $x_{1}, y_{1} \in X_{1}, x_{2}, y_{2} \in X_{2}$; hence, any cut $\delta(S) \in \mathcal{S}$ satisfies these equalities, which implies that it has the claimed form. Say, $d=\sum_{A \in \mathcal{A}} \lambda_{A} \delta\left(A \times X_{2}\right)+\sum_{B \in \mathcal{B}} \lambda_{B} \delta\left(X_{1} \times B\right)$. Then $d_{1}=\sum_{A \in \mathcal{A}} \lambda_{A} \delta(A)$ and $d_{2}=\sum_{B \in \mathcal{B}} \lambda_{B} \delta(B)$. Note that no two cuts $\delta\left(A \times X_{2}\right)$ and $\delta\left(X_{1} \times B\right)$ can be put together in a nested family. Hence, the $m$ nested families partitioning $\mathcal{S}$ yield a partition of the cuts $\delta(A)(A \in \mathcal{A})$ in $p$ nested families and a partition of the cuts $\delta(B)(B \in \mathcal{B})$ in $q$ nested families with $p+q=m$. Therefore, $p \geq \operatorname{dim}\left(X_{1}, d_{1}\right)$ and 
$q \geq \operatorname{dim}\left(X_{2}, d_{2}\right)$, which implies the converse inequality: $\operatorname{dim}(X, d) \geq \operatorname{dim}\left(X_{1}, d_{1}\right)+$ $\operatorname{dim}\left(X_{2}, d_{2}\right)$.

\section{Bounds on the $\ell_{1}$-Dimension}

A metric $d$ is called $\ell_{1}$-rigid if $d$ lies on a simplex face of the cone $\operatorname{CUT}(X)$; this is clearly equivalent to requiring that $d$ has a unique decomposition as (2.1). Then its $\ell_{1}$ dimension is equal to the smallest number of nested families needed to cover the set $\mathcal{S}$ of cuts entering in this decomposition. Several examples of $\ell_{1}$-rigid metric spaces are given in [5] and the behavior of $\ell_{1}$-rigidity under several operations is studied there. In particular, it is shown that the direct product of two metric spaces $\left(X_{1}, d_{1}\right)$ and $\left(X_{2}, d_{2}\right)$ is $\ell_{1}$-rigid if and only if both $\left(X_{1}, d_{1}\right)$ and $\left(X_{2}, d_{2}\right)$ are $\ell_{1}$-rigid. Moreover, all hypercube embeddable graphs are shown to have $\ell_{1}$-rigid path metrics. For instance, for the star $K_{1, n}$ with the set of leaves $\{1, \ldots, n\}$,

$$
\sum_{i=1}^{n} \delta(\{i\})
$$

is the unique decomposition of its path metric as a sum of cuts, called star decomposition. (Remember that a leaf in a graph is a node of degree one.) Thus the $\ell_{1}$-dimension of a hypercube embeddable graph can be determined by examining the decomposition of its path metric as (2.1). This can be easily done in the case of trees. Namely, it follows from the next result that the $\ell_{1}$-dimension of a tree $T$ is equal to $\lceil p / 2\rceil$, where $p$ denotes the number of leaves of $T$.

Lemma 3.1 [9], [6]. The following assertions are equivalent for a tree $T=(V, E)$ :

(i) The path metric of $T$ can be embedded into $\ell_{1}^{m}$.

(ii) $T$ can be covered by $m$ paths.

(iii) $T$ has at most $2 m$ leaves.

In particular, the star $K_{1, n}$ has $\ell_{1}$-dimension $\lceil n / 2\rceil$.

We give below some lower bounds for the parameter $c(m)$ that use a construction based on the star $K_{1, n}$. Note that any star $K_{1, n}$ is an instance of a median space. Remember that a metric space $(X, d)$ is a median space if $|I(x, y) \cap I(x, z) \cap I(y, z)|=1$ for all distinct $x, y, z \in X$; where $I(x, y)=\{u \in X \mid d(x, y)=d(x, u)+d(u, y)\}$ denotes the interval between $x, y \in X$. The following fact is easily established; see [13]. (It follows essentially from the above mentioned rigidity result for the isometric subgraphs of the hypercube and the fact that a median space can be interpreted as the graphic metric space of a hypercube embeddable graph with suitable edge-weights.)

Remark 3.2. Every median space is $\ell_{1}$-rigid.

Proposition 3.3. $\quad c(3) \geq 10 ; c(m) \geq m^{2}$ for $m \geq 5$ odd; $c(m) \geq m^{2}-1$ for $m \geq 4$ even. 
Proof. We start with an observation. Let $(X, d)$ denote the graphic metric space of the graph $G:=K_{1, s} \times K_{1, t}$ (the Cartesian product of the two stars $K_{1, s}$ and $K_{1, t}$ ) where $s, t \geq 3$ are odd. Its $\ell_{1}$-dimension is $\operatorname{dim}(X, d)=\lceil s / 2\rceil+\lceil t / 2\rceil$ (applying Lemmas 3.1 and 2.3, as $K_{1, s}, K_{1, t}$ are trees with $s$ and $t$ leaves, respectively). Say $G$ has node set $X:=V_{1} \times V_{2}$, where $V_{1}:=\left\{u_{0}, u_{1}, \ldots, u_{s}\right\}$ and $V_{2}:=\left\{v_{0}, v_{1}, \ldots, v_{t}\right\}\left(u_{0}, v_{0}\right.$ denoting the respective "centers" of the stars $K_{1, s}$ and $K_{1, t}$ ). Then $d$ can be decomposed (in a unique way) as

$$
d=\sum_{i=1}^{s} \delta\left(S_{i}\right)+\sum_{j=1}^{t} \delta\left(T_{j}\right)
$$

setting $S_{i}:=\left\{u_{i}\right\} \times V_{2}(i=1, \ldots, s)$ and $T_{j}:=V_{1} \times\left\{v_{j}\right\}(j=1, \ldots, t)$. Clearly, any two cuts $\delta\left(S_{i}\right), \delta\left(T_{j}\right)$ do not form a nested pair, while any two $\delta\left(S_{i}\right), \delta\left(S_{j}\right)$ (or $\delta\left(T_{i}\right)$, $\left.\delta\left(T_{j}\right)\right)$ do form a nested pair. This shows again that $\operatorname{dim}(X, d)=\lceil s / 2\rceil+\lceil t / 2\rceil$. On the other hand, if we delete the element $x=\left(u_{i_{0}}, v_{j_{0}}\right)$ from $X$ (where $i_{0} \in\{1, \ldots, s\}$ and $j_{0} \in\{1, \ldots, t\}$ correspond to leaves in the two trees), then the two cuts $\delta\left(S_{i_{0}} \backslash\{x\}\right)$ and $\delta\left(T_{j_{0}} \backslash\{x\}\right)$ become a nested pair. Hence, the cuts $\delta\left(S_{i} \backslash\{x\}\right), \delta\left(T_{j} \backslash\{x\}\right)$ can now be partitioned into $\lceil s / 2\rceil+\lceil t / 2\rceil-1$ nested subfamilies, which shows that

$$
\operatorname{dim}(X \backslash\{x\}, d) \leq\left\lceil\frac{s}{2}\right\rceil+\left\lceil\frac{t}{2}\right\rceil-1
$$

for such $x$.

We can now proceed with the proof. We first show that $c(m) \geq m^{2}$ for $m$ odd. For this, it suffices to construct a metric space $(Y, d)$ such that $|Y| \geq m^{2}, \operatorname{dim}(Y, d) \geq m+1$, and $\operatorname{dim}(Y \backslash\{y\}, d) \leq m$ for all $y \in Y$. Namely, let $(X, d)$ be the graphic metric space of the graph $K_{1, m} \times K_{1, m}$, with $\operatorname{dim}(X, d)=m+1$. Let $Y \subset X$ be a minimal subset of $X$ such that $\operatorname{dim}(Y, d)=m+1$; then $\operatorname{dim}(Y \backslash\{y\}, d) \leq m$ for all $y \in Y$. This metric space $(Y, d)$ does the job as $|Y| \geq m^{2}$ in view of relation (3.2) above. Proceed in the same way to show the inequalities $c(3) \geq 10$ and $c(m) \geq m^{2}-1$ for $m$ even. As to the former, $K_{1, m} \times K_{1, m}$ is replaced by $K_{2} \times K_{5}$, whereas for the latter, the graphic metric space $(X, d)$ of $K_{1, m-1} \times K_{1, m+1}$ with $\operatorname{dim}(X, d)=m+1$ and $(Y, d)$ a subspace such that $\operatorname{dim}(Y, d)=m+1, \operatorname{dim}(Y \backslash\{y\}, d) \leq m$ for all $y \in Y$ are considered.

\section{The $\ell_{1}$-Dimension of $K_{n}$}

Although it was fairly easy to establish the asserted bounds on $c(m)$ in Proposition 3.3 without explicitly knowing the $\ell_{1}$-dimension of the graphic metric space associated to the complete graph $K_{m}$, it is nevertheless of interest to see how many equidistant points the rectilinear space of dimension $m$ can harbor.

It is obvious that at least $2 m$ equidistant points can be placed in the $m$-dimensional rectilinear space; namely, consider the coordinate vectors $\pm e_{i}(i=1, \ldots, m)$. We refer to the configuration of points $\pm e_{i}(i=1, \ldots, m)$ as to the trivial embedding of the complete graph $K_{m}$ (up to a multiplicative factor). Is it the best that can be done? This question was already raised by R.K. Kusner (see Problem 0 of [8]). For related problems see [1] and [7]. The answer is yes in dimension $m \leq 3$ but is not known for $m>3$. We 
conjecture that the answer is always yes; in other words, that

$$
\operatorname{dim}\left(K_{2 m+1}\right)=\operatorname{dim}\left(K_{2 m+2}\right)=m+1 \quad \text { for all } \quad m \geq 1 .
$$

(For brevity, we let $\operatorname{dim}\left(K_{m}\right)$ denote the $\ell_{1}$-dimension of the graphic metric space attached to $K_{m}$.) The case $m=1$ is obvious and the case $m=2$ follows from the following easy argument. Assume that $u_{i} \in \mathbb{R}^{2}(i=1,2,3,4,5)$ are five equidistant points in the plane. Let $X \subseteq\left\{u_{1}, u_{2}, u_{3}, u_{4}, u_{5}\right\}$ consist of the points corresponding to the minimum and maximum values in each of the two coordinate directions; so $|X| \leq 4$. The points in $X$ determine a rectangle which encloses all $u_{i}$ 's. It is easy to see that this rectangle is covered by the intervals $I(x, y)$ for $x, y \in X$. Therefore, some $u_{i}$ lies in an interval $I\left(u_{j}, u_{k}\right)$, which yields a contradiction. Moreover, a configuration of four equidistant points in the plane is (up to translation) the trivial one. On the other hand, $K_{3}$ has an infinity of embeddings in $\mathbb{R}^{2}$ (e.g., by the points $(1,0),(0,1)$, and $(-a, a-1)$ for $0 \leq a \leq 1)$. However, all of them correspond to the star decomposition (3.1). Indeed, if $x_{1}, x_{2}, x_{3} \in \mathbb{R}^{2}$ realize an embedding of $K_{3}$, then $x_{0}, x_{1}, x_{2}, x_{3}$ realize an embedding of the star $K_{1,3}$ (up to a factor of $\frac{1}{2}$ ), where $x_{0}$ is the median point of $x_{1}, x_{2}, x_{3}$ in $\mathbb{R}^{2}$.

The case $m=3$ is settled in the next result.

Proposition 4.1. $\operatorname{dim}\left(K_{7}\right)=\operatorname{dim}\left(K_{8}\right)=4$.

Proof. What we have to show is that any set $X \subseteq \mathbb{R}^{3}$ of equidistant points has cardinality $|X| \leq 6$. Denote the $\ell_{1}$-distance between two points $x, y$ by $d(x, y)$ and assume that $d(x, y)=1$ for all $x \neq y \in X$. Let $u \neq v \in X$ for which the interval $I(u, v)$ has minimum dimension $k$. Suppose first that $k=1$; that is, $I(u, v)$ is a segment. Then $X \backslash\{u, v\}$ lies in the hyperplane orthogonal to the segment $I(u, v)$ and passing through its midpoint. Therefore, $|X \backslash\{u, v\}| \leq 4$ (as $X \backslash\{u, v\}$ is a two-dimensional equidistant set of points), which implies that $|X| \leq 6$. Hence, we can assume from now on that $k=2,3$; that is, $I(u, v)$ is a rectangle or a parallelepiped. Let $\varphi(x)$ denote the median of $x, u$, and $v$ (with the $i$ th component the medium value of $x_{i}, u_{i}$, and $v_{i}$ ). In other words, $\varphi(x)$ is the projection of $x$ onto $I(u, v)$. We have $d(x, \varphi(x))=d(u, \varphi(x))=d(v, \varphi(x))=\frac{1}{2}$ for all $x \in X \backslash\{u, v\}$. We distinguish two cases, depending on whether the medians $\varphi(x)$ are all equal or not.

Suppose first that all medians $\varphi(x)(x \in X \backslash\{u, v\})$ coincide. Then the points in $X$ together with the common median $\varphi(x)$ form an embedding of the star $K_{1,|X|}$ in the rectilinear 3-space. By Lemma 3.1, this implies that $|X| \leq 6$.

We can henceforth assume that there exist at least two distinct medians. We show that, in fact, $|X| \leq 4$ holds. To fix ideas, we assume that $u_{i} \leq v_{i}$ for all $i=1,2,3$. Hence, $\varphi(x)_{i}=x_{i}$ if $u_{i}<x_{i}<v_{i}, \varphi(x)_{i}=u_{i}$ if $x_{i} \leq u_{i}$, and $\varphi(x)_{i}=v_{i}$ if $x_{i} \geq v_{i}$. A first useful observation is that some half-plane orthogonal to a coordinate axis separates $x$ and $y$ from $I(u, v)$ if $\varphi(x) \neq \varphi(y)$. Namely,

(a) if $\varphi(x) \neq \varphi(y)$, then $x_{i}, y_{i}<u_{i}$ or $x_{i}, y_{i}>v_{i}$ for some $i=1,2,3$.

(For, if not, then $\varphi(x)$ would belong to the interval $I(x, y)$ which would imply that $\varphi(x)=\varphi(y)$, a contradiction.) In particular, this implies that any two distinct medians $\varphi(x), \varphi(y)$ lie on a common facet of $I(u, v)$ when $I(u, v)$ has dimension $k=3$. A 


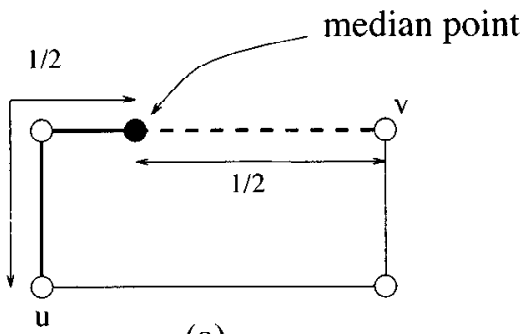

(a)

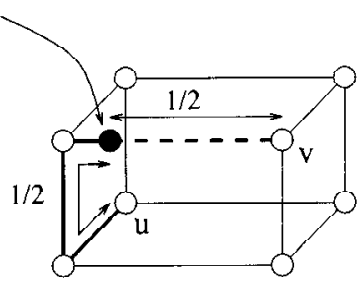

(b)

Fig. 1. The interval $I(u, v)$ : (a) $k=2$, (b) $k=3$.

second observation is that

(b) no two distinct medians $\varphi(x), \varphi(y)$ lie on a common edge of $I(u, v)$.

(This follows easily from the fact that both $\varphi(x)$ and $\varphi(y)$ are at the same distance $\frac{1}{2}$ from $u$ and $v$; see Fig. 1 for an illustration.) This permits us to derive that any two medians are distinct:

(c) $\varphi(x) \neq \varphi(y)$ for all $x \neq y \in X \backslash\{u, v\}$.

Indeed, if $\varphi(x)=\varphi(y)$, then $\varphi(x)$ lies in the interval $I(x, y)$, as $d(x, \varphi(x))=\varphi(y, \varphi(x))$ $=\frac{1}{2}$. Moreover, $x_{i} \leq u_{i}$ (resp. $x_{i} \geq v_{i}$ ) if and only if $y_{i} \leq u_{i}$ (resp. $y_{i} \geq v_{i}$ ). Hence, $\max \left(x_{i}, y_{i}\right)=u_{i}$ whenever $x_{i} \leq u_{i}$ and $\min \left(x_{i}, y_{i}\right)=v_{i}$ whenever $x_{i} \geq v_{i}$. Let $\varphi(z)$ be a second median which is distinct from $\varphi(x)=\varphi(y)$. By applying (a) to both pairs $x, z$ and $y, z$, we can find two distinct indices $i, j$ for which $\varphi(z)_{i}=\varphi(x)_{i}, \varphi(z)_{j}=\varphi(x)_{j}$. Therefore, $\varphi(z)$ and $\varphi(x)$ lie on a common edge of $I(u, v)$, which contradicts (b).

Some final observations follow. No median $\varphi(x)$ lies in the interior of $I(u, v)$ if $I(u, v)$ has dimension $k=3$ (else, $x=\varphi(x) \in I(u, v)$ ). Moreover, no two distinct medians $\varphi(x), \varphi(y)$ lie in the interior of $I(u, v)$ if $k=2$ or in the interior of a facet of $I(u, v)$ if $k=3$ (else, $d(x, y)=d(\varphi(x), \varphi(y))<1$ ). If there is a median $\varphi(x)$ lying in the interior of an edge of $I(u, v)$ incident with $u$ or $v$ (thus, an arbitrary edge in the case $k=2$ ), then the other edges incident with this edge contain no median (because the distance of a median to both $u$ and $v$ is equal to $\frac{1}{2}$; see Fig. 1).

We can now conclude that $|X \backslash\{u, v\}| \leq 2$; that is, $|X| \leq 4$. This follows easily from the above observations if there is no median lying in the interior of $I(u, v)$ when $k=2$, or in the interior of a facet of $I(u, v)$ when $k=3$. We now consider the case when some median $\varphi(x)$ lies in the interior of a rectangle $R$ which is either $I(u, v)$ when $k=2$, or a facet of $I(u, v)$ when $k=3$. Then all other medians lie on the boundary of $R$ and in fact there are at most two of them. We assume that there exist two medians $\varphi(y)$ and $\varphi(z)$ on the boundary of $R$. Now all points $a \in X \backslash\{u, v\}$ lie on the same side of the hyperplane $H$ determined by $R$ (else, $d(a, b)=d(a, \varphi(a))+d(\varphi(a), \varphi(b))+d(\varphi(b), b)>1$ if $a$ and $b$ are on distinct sides of $H$ ). Let $H^{\prime}$ and $H^{\prime \prime}$ denote the two other hyperplanes parallel to the coordinate hyperplanes and passing through $\varphi(x)$. So, $\ell:=H^{\prime} \cap H^{\prime \prime}$ is the line through $x$ and $\varphi(x)$. Clearly, $\varphi(y)$ and $\varphi(z)$ are separated by both hyperplanes $H^{\prime}$ and $H^{\prime \prime}$ and thus the same holds for $y$ and $z$. Let $w$ denote the median of $x, y$, and 

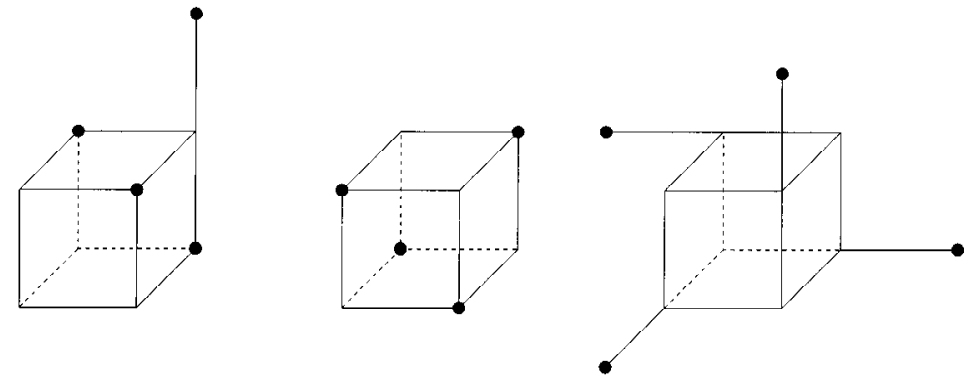

Fig. 2. Some embeddings (up to scale) of $K_{4}$ in $\mathbb{R}^{3}$.

$z$. Since each closed half-space determined by $H^{\prime}$ and $H^{\prime \prime}$ contains $x$ and one of $y, z$, necessarily $w$ belongs to $H^{\prime} \cap H^{\prime \prime}=\ell$; that is, $w \in[x, \varphi(x)]$. Therefore, $w=\varphi(x)$ (as $\left.d(w, x)=d(\varphi(x), x)=\frac{1}{2}\right)$. This implies that $\varphi(x)=\varphi(y)=\varphi(z)$, a contradiction.

The proof of Proposition 4.1 establishes, in fact, the following result about the possible $\ell_{1}$-embeddings of $K_{5}$ and $K_{6}$ in three-dimensional space.

Corollary 4.2. Any embedding of $K_{5}$ in $\mathbb{R}^{3}$ corresponds to the star embedding (3.1) and $K_{6}$ has a unique (up to translation) embedding in $\mathbb{R}^{3}$ (the trivial one).

Proof. Let $X \subseteq \mathbb{R}^{3}$ be a set of equidistant points with pairwise distances 1 . If $|X|=$ 5,6 , then, by the proof of Proposition 4.1, all medians $\varphi(x)(x \in X \backslash\{u, v\})$ coincide with, say, $x_{0} \in \mathbb{R}^{3}$. Therefore, $X \cup\left\{x_{0}\right\}$ is an embedding of the $\operatorname{star} K_{1,|X|}$ in $\mathbb{R}^{3}$. If $|X|=5$, the result follows from the fact that the path metric of a star is $\ell_{1}$-rigid. Now, let $|X|=6$. Up to translation, we can assume that $x_{0}=0:=(0,0,0)$. We show that $X=\left\{ \pm \frac{1}{2} e_{i} \mid i=1,2,3\right\}$. Indeed, each of the six open half-spaces supported by the coordinate hyperplanes through the origin cannot contain more than one point from $X \backslash\{0\}$ since 0 does not belong to the interval of any two points from any of these open half-spaces. Consequently, all six points from $X \backslash\{0\}$ lie on the coordinate axes, whence the result follows.

We conclude with some examples of embeddings (up to scale) of $K_{4}$ in the space $\mathbb{R}^{3}$. The embeddings shown in Fig. 2 correspond, respectively, to the star decomposition $\sum_{i=1}^{4} \delta(\{i\})$ and the following two decompositions: $\sum_{i=2}^{4} \delta(\{1, i\})$ and $\sum_{i=1}^{4} \delta(\{i\})+$ $\sum_{i=2}^{4} \delta(\{1, i\})$. The first two give an embedding of $K_{4}$ up to a factor of 2 and the last one up to a factor of 4 .

\section{Acknowledgment}

We thank the referees for providing the references to the work of Bonan-Hamada and Guy. 


\section{References}

1. K. Ball, Isometric embedding in $l_{p}$-spaces, European J. Combin. 11 (1990), 305-311.

2. H.-J. Bandelt and V. Chepoi, Embedding metric spaces in the rectilinear plane: a six-point criterion, Discrete Comput. Geom. 15 (1996), 107-117.

3. H.-J. Bandelt and A.W.M. Dress, A canonical decomposition theory for metrics on a finite set, Adv. in Math. 92 (1992), 47-105.

4. E.K. Bonan-Hamada, A bounded compactness theorem for $l^{1}$ embeddability of metric spaces in $\mathbb{R}^{2}, \mathrm{Ph} . \mathrm{D}$. thesis, Colorado University, Boulder, 1996.

5. M. Deza and M. Laurent, $\ell_{1}$-Rigid graphs, J. Alg. Combin. 3 (1994), 153-175.

6. B. Fichet, Dimensionality problems in $L_{1}$-norm representations, in Classification and Dissimilarity Analysis, Lecture Notes in Statistics, Vol. 93, Springer-Verlag, Berlin, 1994, pp. 201-224.

7. Z. Füredi, J.C. Lagarias, and F. Morgan, Singularities of minimal surfaces and networks and related extremal problems in Minkowski space, in Discrete and Computational Geometry (J.E. Goodman, R. Pollack, and W. Steiger, eds.), DIMACS Series in Discrete Mathematics and Theoretical Computer Science, Vol. 6, 1991, pp. 95-109.

8. R.K. Guy, An olla-podrida of open problems, often oddly posed, Amer. Math. Monthly 90 (1983), 196-199.

9. F. Hadlock and F. Hoffman, Manhattan trees, Utilitas Math. 13 (1978), 55-67.

10. A.V. Karzanov, Metrics and undirected cuts, Math. Programming 32 (1985), 183-198.

11. S.M. Malitz and J.I. Malitz, A bounded compactness theorem for $L^{1}$-embeddability of metric spaces in the plane, Discrete Comput. Geom. 8 (1992), 373-385.

12. K. Menger, Untersuchungen über allgemeine Metrik, Math. Ann. 100 (1928), 75-163.

13. M.L.J. van de Vel, Theory of Convex Structures, Elsevier/North-Holland, Amsterdam, 1993.

Received March 19, 1996, and in revised form March 14, 1997. 